\title{
Aspectos relacionados à alta precoce do Recém-Nascido: revisão integrativa
}

\author{
Aspects related to the early discharge of the Newborn: integrative review
}

Aspectos relacionados a la alta precoce delo Recién Nacido: revisión integrativa

Isabela Miranda Queiroz ${ }^{1 *}$, Bernardo Cordeiro Castro¹, Evandro Barbosa Dos Anjos¹, Jéssica Velloso Fargnoli Braga², Ana Luiza Fonseca Maia Caetano', Ivan Ivens Brito'

\section{RESUMO}

Objetivo: Considerando o impacto do tempo de permanência hospitalar nos cuidados maternos e na saúde do recém-nascido, este estudo visa identificar as atualizações dos aspectos relacionados à alta precoce do neonato proveniente de gestação e parto não complicado. Método: Trata-se de uma revisão integrativa, de publicações científicas de 2008 a 2017, indexadas nas bases de dados: Biblioteca Eletrônica Científica Online no inglês (Scielo), Literatura Latino-Americana e do Caribe em Ciências da Saúde (LILACS) e no Sistema Online de Busca e Análise de Literatura Médica (Medline). Resultado: Na busca inicial foram identificados 816 artigos, dos quais 10 foram selecionados para análise na íntegra. Na literatura não há consenso na definição de alta precoce e seu seguimento. Alguns estudos apontam menor risco de readmissão em a termos saudáveis com permanência hospitalar reduzida se associada a um programa de acompanhamento e possível benefício na internação mais prolongada em prematuros tardios. Ainda assim, as evidências disponíveis não são suficientes para rejeitar ou apoiar a prática. Considerações finais: Os aspectos relacionados à alta do recém-nascido devem ser avaliados individualmente. Além disso, percebe-se que no Brasil não há estudos que avaliem o impacto da alta precoce na saúde do recém-nascido.

Palavras-chave: Recém-nascido, Alta do paciente, Tempo de internação, Cuidado Pós-Natal, Readmissão do paciente.

\begin{abstract}
Aims: Considering the impact of hospital stay time on maternal care and newborn health, this study aims to identify the updates related to early discharge from the newborn from uncomplicated gestation and delivery. Method: This is an integrative review of scientific publications from 2008 to 2017, indexed in the databases: Scientific Electronic Library Online (SciELO), Latin American and Caribbean Health Sciences Literature (LILACS), in Medical Literature Analysis and Retrieval System Online (Medline). Result: In the initial search, 816 articles were identified, of which 10 were selected for full analysis. In the literature there is no consensus on the definition of early discharge and it's follow-up. Some studies point a lower risk of readmission in healthy term infants with reduced hospital stay if associated with a follow-up program and possible benefit in longer hospitalization in post-term neonates. Still, the evidences are not sufficient to reject or support the practice. Final considerations: The aspects of discharge of the newborn should be valued individually. Besides, in Brazil there are no studies that apprise the impact of early discharge in the newborn health.
\end{abstract}

Keywords: Newborn, Patient Discharge, Length of Stay, Postnatal Care, Patient Readmission.

\footnotetext{
${ }^{1}$ Faculdades Integradas do Norte de Minas (Funorte). Montes Claros - MG. *E-mail: isabelama@yahoo.com.br ${ }^{1}$ Faculdades Integradas Pitágoras (FIP-MOC). Montes Claros - MG.
} 


\section{RESUMEN}

Objetivo: Teniendo en cuenta el impacto de la duración de la hospitalización en la atención materna y la salud del recién nacido, este estudio tiene como objetivo identificar actualizaciones sobre aspectos relacionados con el alta temprana del embarazo y el parto sin complicaciones. Método: Esta es una revisión integradora de publicaciones científicas de 2008 a 2017, indexada en las bases de datos: Biblioteca Electrónica Científica en Línea (Scielo), Literatura de Ciencias de la Salud de América Latina y el Caribe (LILACS) y Sistema de búsqueda y análisis de literatura médica en línea (Medline). Resultado: En la búsqueda inicial, se identificaron 816 artículos, de los cuales 10 fueron seleccionados para análisis completo. No existe consenso en la literatura sobre la definición de alta temprana y su seguimiento. Algunos estudios indican menor riesgo de reingreso en términos saludables con estadía hospitalaria reducida asociada con un programa de seguimiento y posible beneficio en hospitalización más prolongada en neonatos prematuros tardíos. Pero la evidencia disponible no es suficiente para rechazar o apoyar la práctica. Consideraciones finales: Los aspectos relacionados con el alta del neonato deben considerarse individualmente. Además, está claro que en Brasil no hay estudios que evalúen el impacto del alta temprana en la salud del recién nacido.

Palabras Clave: Recién nacido, Alta del paciente, Tiempo de permanência, Atención Posnatal, Readmisión del paciente.

\section{INTRODUÇÃO}

A hospitalização devido ao parto se tornou uma norma desde 1940, e é um dos motivos mais frequentes de admissão hospitalar em países industrializados. Desde seu estabelecimento até hoje, a permanência hospitalar sofreu diversas alterações em todo o mundo. Vários estudos demonstram que a duração da estadia tem diminuído nas últimas décadas na tentativa de reduzir os custos e aumentar o bem-estar da mulher. $\mathrm{Na}$ década de 50 , a permanência era de seis a quatorze dias; na década de 70 , foi reduzida para seis dias; já no final dos anos 90, passou-se para 48 horas (JAMES L, et al., 2017; METCALFE A, et al., 2016).

Estudos subseqüentes observaram que um tempo de permanência hospitalar mais curto pode aumentar o risco de icterícia, desidratação, infecções, complicações gastrointestinais e dificuldades de alimentação. Outros problemas apontados foram a dificuldade em iniciar e manter a amamentação por falta de apoio à prática e a deficiência no atendimento de acompanhamento pós natal (JACKSON GL, 2000; ZIMMERMAN DR, 2003; CHANG RKR 2008; LUCIANO R, 2015).

Além da diminuição dos custos, um tempo de internação mais curto é apontado como contribuinte para a melhoria da relação mãe e filho, bem como melhores taxas de amamentação devido ao ambiente familiar. As desvantagens incluiriam menor tempo para ensinar sobre cuidados com o bebê, para iniciar e estabelecer a prática da amamentação; e menor oportunidade de identificar problemas latentes na mãe e no lactente, bem como o maior uso de testes de triagem neonatal (BRASIL, 2016; METCALFE A, et al., 2016).

Alguns estudos epidemiológicos americanos (PAUL IM, 2006; WATT S, 2005; DATAR A, 2006) avaliaram a qualidade do tempo de internação nos recém-nascidos através das taxas de readmissão. Entre eles, as readmissões após a alta precoce variaram de sem aumento para um aumento significativo. A não padronização nos conceitos de alta precoce, seguimento e momento das readmissões, no entanto, dificulta a comparação dos resultados tornando as análises inconclusivas (BENITZ WE, 2015).

A definição de alta precoce varia de acordo com o país e entre hospitais, não permitindo, portanto, um consenso em relação a esse conceito. A literatura internacional cita, como tempo de permanência hospitalar, tanto 24 horas, quanto 48 e 72 horas. Normalmente, estabelece-se o tempo entre 24 e 48 horas após o nascimento, sempre com recém-nascido $(R N)$ a termo, proveniente de gestação e parto não complicados. Já a alta muito precoce seria aquela realizada antes das 24 horas (JAMES L, et al., 2017; METCALFE A, et al., 2016; GRACIA SR, et al., 2017). 
No Brasil, por sua vez, não existe uma definição oficial sobre o tempo de permanência hospitalar pósparto. A Portaria 2.068 do Ministério da Saúde, publicada no Diário Oficial da União, de 21 de outubro de 2016, recomenda que a permanência hospitalar não seja inferior a 24 horas devido à importância desse período para educação da mãe e detecção de patologias neonatais (BRASIL, 2016).

Os últimos trabalhos aconselham uma decisão conjunta entre a mãe e profissionais em que prevaleça as necessidades da mulher. Um tempo de estadia hospitalar específico para cada recém-nascido a depender de sua estabilidade e saúde da mãe, da rede de apoio para o cuidado em casa e suporte no acompanhamento (SILVA HMCPP, 2017; LEMYRE B, 2018).

Considerando o impacto do tempo de permanência hospitalar nos cuidados maternos e na saúde do RN, este estudo visa identificar as atualizações dos aspectos relacionados à alta precoce do neonato proveniente de parto e gestação não complicados.

\section{MÉTODOS}

Trata-se de uma revisão integrativa da literatura sobre os aspectos relacionados à alta precoce do RN a termo. Para atingir o objetivo, foi realizada busca nas bases eletrônicas: Online de Busca e Análise de Literatura Médica (Medline), Literatura Latino-Americana e do Caribe em Ciências da Saúde (LILACS) e Scientific Eletronic Library Online (SciELO), no período de julho a agosto de 2017, utilizando-se os descritores "Patient Discharge", "Length Of Stay", "Post natal Care", "Patient Readmission" e relacionando-os a "Infant, Newborn".

Foram definidos, como critérios de inclusão: artigos publicados nos últimos dez anos, que correspondem aos anos de 2008 a 2017; nos idiomas inglês, espanhol ou português; e que tratavam dos temas "alta precoce do recém-nascido (RN)", "reinternação" e "cuidados pós-natal".

Os critérios de exclusão levaram em consideração os artigos que abordavam unicamente RN pré-termo e parto cesáreo, bem como os que tratavam de pacientes com complicações gestacionais e periparto, além de artigos repetidos, não acessíveis em texto completo e que não se ocupavam diretamente do tema deste estudo.

Todos os títulos disponíveis foram avaliados. Em seguida, os resumos dos artigos selecionados foram examinados para adequação aos critérios de inclusão e exclusão. Os artigos foram estudados para relevância do conteúdo. Após a seleção, iniciou-se a análise crítica do material que permitiu identificar os seguintes eixos temáticos: critérios de alta precoce, vantagens e desvantagens da alta precoce, influência da mesma nas reinternações dos $\mathrm{RN}$ a termo e a importância do segmento pós-alta.

\section{RESULTADOS e DISCUSSÃO}

A amostra inicial desta pesquisa foi constituída por 873 artigos, dos quais, após considerados os critérios de exclusão, dez foram submetidos à avaliação detalhada (Tabela 1) para extração e análise dos dados. Foram incluídos três documentos considerados relevantes ao estudo, independentes da busca nas bases de dados: as portarias ํㅜ 1.130, de 5 de agosto de 2015 e ํo 2.068 de 21 de outubro de 2016, ambas do Ministério da Saúde, e o Guidelines for Perinatal Care, do Colégio Americano de Ginecologia e Obstetrícia.

A relevância do tempo entre o nascimento e a alta do RN está na identificação das complicações para mãe e para o RN, além de ser um período essencial para a prestação tanto de apoio, quanto de informações à mãe e à família (BRAVO P, et al., 2011).

Tem-se discutido muito sobre a alta precoce nas últimas décadas, mas apesar dos estudos demonstrarem o seu benefício, como a satisfação materna e familiar e a redução dos custos, ainda não se pode afirmar que não há consequências negativas que superem as suas vantagens (JAMES L, et al., 2017). Além disso, não existe consenso em relação à definição da alta precoce e seguimento após a alta, o que dificulta a avaliação dos resultados das pesquisas. 
Tabela 1 - Artigos selecionados para revisão sobre alta precoce.

\begin{tabular}{|c|c|c|c|}
\hline Autor (es) & Ano & Título & Conclusão \\
\hline $\begin{array}{l}\text { Bravo P, Uribe } \mathrm{C} \text {, } \\
\text { Contreras } \mathrm{A} \text {. }\end{array}$ & 2011 & $\begin{array}{l}\text { Alta hospitalar pós-natal precoce: as } \\
\text { consequências da redução do tempo de } \\
\text { permanência de mulheres e recém- } \\
\text { nascidos }\end{array}$ & $\begin{array}{l}\text { A evidência disponível não é suficiente para rejeitar ou apoiar a prática de alta precoce } \\
\text { pós-natal; diferentes estudos relataram resultados diferentes para mulheres e recém- } \\
\text { nascidos. Discute-se a necessidade de uma pesquisa clínica sistemática. }\end{array}$ \\
\hline $\begin{array}{l}\text { James L, Sweet L, } \\
\text { Donnellan-Fernandez } \\
\mathrm{R}\end{array}$ & 2016 & $\begin{array}{l}\text { Iniciação e apoio ao aleitamento } \\
\text { materno: uma revisão da literatura } \\
\text { sobre o valor das mulheres e o impacto } \\
\text { da alta precoce }\end{array}$ & $\begin{array}{l}\text { Os resultados sugerem que o tempo de permanência pós-natal individualizado pode } \\
\text { ser benéfico para iniciar a amamentação. Cinco valores não foram afetados pela alta } \\
\text { precoce, mas sim pela prática individual das parteiras. Há consenso na literatura de } \\
\text { que a alta precoce promoveu um ambiente confortável para apoiar o início da } \\
\text { amamentação. Amplas variações na definição da alta pós-natal tornaram difícil tirar } \\
\text { conclusões influentes. Portanto, mais pesquisas são necessárias. }\end{array}$ \\
\hline $\begin{array}{l}\text { Rite Gracia R, } \\
\text { Muñuzuri } A \text {, López } E \text {, } \\
\text { et al. }\end{array}$ & 2016 & $\begin{array}{l}\text { Critérios para alta hospitalar do recém- } \\
\text { nascido a termo saudável após o parto }\end{array}$ & $\begin{array}{l}\text { Pode-se considerar, em recém-nascidos a termo saudáveis, uma permanência } \\
\text { hospitalar reduzida (menos de } 48 \text { horas após o nascimento), mas isso não é apropriado } \\
\text { para todas as mães e todos os recém-nascidos. Os recém-nascidos que recebem alta } \\
\text { antes das } 48 \text { horas do nascimento devem ser avaliados entre o terceiro e o quarto dia } \\
\text { de vida. }\end{array}$ \\
\hline $\begin{array}{l}\text { De Carolis M, Cocca C, } \\
\text { Valente } \mathrm{E} \text {, et al }\end{array}$ & 2014 & $\begin{array}{l}\text { Programa de acompanhamento } \\
\text { individualizado e alta precoce em } \\
\text { recém-nascidos a termo }\end{array}$ & $\begin{array}{l}\text { A alta precoce em associação com um programa de acompanhamento individualizado } \\
\text { resultou segura para o recém-nascido e eficaz para o início e a duração da } \\
\text { amamentação }\end{array}$ \\
\hline $\begin{array}{l}\text { Elisabeth Kurth E, } \\
\text { Krähenbühl K, Eicher } \\
M\end{array}$ & 2016 & $\begin{array}{l}\text { Início seguro em casa: o que os pais de } \\
\text { recém-nascidos precisam após a alta } \\
\text { hospitalar - um estudo em grupo focal }\end{array}$ & $\begin{array}{l}\text { Após a alta hospitalar, os novos pais precisam de apoio prático, acompanhamento e } \\
\text { cuidados. Esse apoio é importante para a saúde e o bem-estar da mãe e do filho. Os } \\
\text { serviços integrados de atendimento, incluindo visitas domiciliares profissionais e linhas } \\
\text { de atendimento } 24 \text { horas, podem ajudar a atender às necessidades de novas famílias. }\end{array}$ \\
\hline
\end{tabular}

REAS/EJCH | Vol.Sup.34 | e1338 | DOI: https://doi.org/10.25248/reas.e1338.2019 Página 4 de 8 


\begin{tabular}{|c|c|c|c|}
\hline $\begin{array}{l}\text { Harron } \mathrm{K}, \text { Gilbert } \mathrm{R}, \\
\text { Cromwell } \mathrm{D} \text {, et al }\end{array}$ & 2017 & $\begin{array}{l}\text { Tempo de internação do recém-nascido } \\
\text { e risco de readmissão }\end{array}$ & $\begin{array}{l}\text { O tempo de internação mais longo do recém-nascido pode beneficiar recém-nascidos } \\
\text { prematuros tardios, possivelmente devido ao aumento do apoio psicossocial ou médico } \\
\text { para aqueles com maior risco de reinternações potencialmente evitáveis após o } \\
\text { nascimento. Pesquisas baseadas em dados observacionais para avaliar as relações } \\
\text { entre o tempo de internação hospitalar e a readmissão devem usar métodos para } \\
\text { reduzir o impacto do confundimento não medido. }\end{array}$ \\
\hline $\begin{array}{l}\text { Metcalfe } A \text {, Mathai } M \text {, } \\
\text { Liu S, et al }\end{array}$ & 2016 & $\begin{array}{l}\text { Proporção de readmissão neonatal } \\
\text { atribuída ao tempo de permanência no } \\
\text { parto: um estudo coorte de base } \\
\text { populacional }\end{array}$ & $\begin{array}{l}\text { Padrões de readmissão sugerem que as taxas de internação são mais baixas após uma } \\
\text { estadia de } 1 \text { a } 2 \text { dias após um parto vaginal e uma permanência de } 2 \text { a } 4 \text { dias após o } \\
\text { parto cesáreo, dado o apoio ambulatorial na comunidade. }\end{array}$ \\
\hline $\begin{array}{l}\text { Lain S, Christine L. } \\
\text { Roberts, Jennifer R. }\end{array}$ & 2015 & $\begin{array}{l}\text { Alta precoce de bebês e risco de } \\
\text { readmissão por icterícia }\end{array}$ & $\begin{array}{l}\text { Este estudo pode informar diretrizes ou políticas sobre a identificação de crianças com } \\
\text { risco de readmissão por icterícia e garantir que o acompanhamento adequado após a } \\
\text { alta seja recebido. }\end{array}$ \\
\hline $\begin{array}{l}\text { Delgado M, Furzán J, } \\
\text { et al. }\end{array}$ & 2013 & $\begin{array}{l}\text { Alta neonatal precoce e consulta } \\
\text { imediata de acompanhamento }\end{array}$ & $\begin{array}{l}\text { Uma fração significativa de recém-nascidos que recebem alta antes de } 48 \text { horas não } \\
\text { atende aos critérios mínimos para alta precoce. O cumprimento da consulta de } \\
\text { acompanhamento é baixo e não se relaciona com o risco individual da criança. As } \\
\text { barreiras específicas que interferem com o cuidado pós-natal imediato do recém- } \\
\text { nascido exposto à alta precoce devem ser definidas. }\end{array}$ \\
\hline $\begin{array}{l}\text { Asensi Monzó } \mathrm{M} \text {, } \\
\text { Fabregat Ferrer } \mathrm{E} . \\
\text { Gutiérrez Singler D, et } \\
\text { al. }\end{array}$ & 2014 & $\begin{array}{l}\text { Atenção nas primeiras } 48 \text { horas após a } \\
\text { alta da maternidade no consultório de } \\
\text { enfermagem ou pediatria }\end{array}$ & $\begin{array}{l}\text { A partir do momento em que o recém-nascido é liberado da maternidade até ser } \\
\text { atendido pela primeira vez pela equipe de pediatra-enfermeiro, passa um importante } \\
\text { período de tempo. }\end{array}$ \\
\hline
\end{tabular}

Fonte: Queiroz IM, et al., 2019.

REAS/EJCH | Vol.Sup.34 | e1338 | DOI: https://doi.org/10.25248/reas.e1338.2019 Página 5 de 8 
A internação hospitalar deve durar tempo necessário para identificar problemas e permitir que a mãe esteja recuperada e preparada para cuidar dela e do seu filho. Tendo isso em vista, a American Academy of Pediactrics estabeleceu critérios de alta precoce, assim como o Ministério da Saúde (Quadro 1). A negligência em relação aos critérios de alta pode implicar em um aumento do risco para o $\mathrm{RN}$ e provocar sua reinternação hospitalar (GRACIA SR, et al., 2017).

Quadro 1 - Critérios de alta hospitalar (simplificados).

\begin{tabular}{|c|c|c|}
\hline & American Academy of Pediactrics & Ministério da Saúde \\
\hline a) & $\begin{array}{l}\text { Sinais vitais do RN estáveis e normais nas } \\
12 \text { horas antes da alta; }\end{array}$ & Em relação ao $\mathrm{RN}$ : \\
\hline b) & $\begin{array}{l}\text { Pelo menos um episódio de evacuação e } \\
\text { micção espontâneas; }\end{array}$ & $\begin{array}{l}\text { i. A termo e com peso adequado para a idade } \\
\text { gestacional, sem comorbidades e com } \\
\text { exame físico normal. }\end{array}$ \\
\hline c) & Pelo menos duas mamadas efetivas; & $\begin{array}{l}\text { ii. } \begin{array}{l}\text { Com ausência de icterícia nas primeiras } 24 \\
\text { horas de vida; }\end{array}\end{array}$ \\
\hline d) & $\begin{array}{l}\text { Não ter nenhuma anormalidade no exame } \\
\text { físico que necessite de continuar internado; }\end{array}$ & Com avaliação de icterícia \\
\hline e) & $\begin{array}{l}\text { Observar icterícia e manejar de acordo com } \\
\text { a gravidade; }\end{array}$ & $\begin{array}{ll}\text { iv. } & \text { Apresentando diurese e evacuação } \\
& \text { espontâneas e controle térmico adequado }\end{array}$ \\
\hline f) & $\begin{array}{l}\text { A mãe apresentar conhecimento, habilidade } \\
\text { e confiança para promover cuidado com o } \\
\mathrm{RN} \text {; }\end{array}$ & $\begin{array}{l}\text { v. Com sucção efetiva, salvo em situações em } \\
\text { que há restrições ao aleitamento materno; }\end{array}$ \\
\hline g) & $\begin{array}{l}\text { Exames laboratoriais do } \mathrm{RN} \text { e da puérpera } \\
\text { revisados; }\end{array}$ & \multirow{4}{*}{$\begin{array}{l}\text { Em uso de substituto do leite } \\
\text { humano/fórmula láctea para situações nas } \\
\text { quais a amamentação é contraindicada, de } \\
\text { acordo com atualização da Organização } \\
\text { Mundial da Saúde OMS/2009 "Razões } \\
\text { médicas aceitáveis para uso de substitutos } \\
\text { do leite". }\end{array}$} \\
\hline h) & Vacina de hepatite B administrada; & \\
\hline i) & $\begin{array}{l}\text { Realização dos exames de rastreamento: } \\
\text { pezinho, orelhinha e olhinho; }\end{array}$ & \\
\hline j) & $\begin{array}{l}\text { Caso a criança apresente riscos sociais a } \\
\text { alta deve ser retardada. }\end{array}$ & \\
\hline
\end{tabular}

Fonte: Queiroz IM, et al., 2019 (Baseado em Kilpatrick SJ et al., 2017; BRASIL, 2016).

Umas das formas mais utilizadas para avaliar a efetividade da alta precoce é a taxa de reinternação. As principais causas de reinternação são icterícia, problemas relacionados à desidratação, perda de peso, hipernatremia, problemas com amamentação e sepse (DE CAROLIS MP, et al., 2014; KURTH E, et al., 2016).

Em estudo realizado na Inglaterra, entre abril de 2005 e fevereiro de 2014, o tempo de permanência para partos vaginais diminuiu $2 \%$ ao ano, de 1,4 dias para 1,2 dias, independente da idade gestacional. Durante 0 mesmo período, o risco de readmissão elevou $4,4 \%$ ao ano, um aumento maior em curto prazo e em prematuros tardios (nascidos entre 34 e 36 semanas). Logo, foi constatado que para cada dia adicional na internação de RN nascidos de parto vaginal, houve um aumento de $3 \%$ no risco de readmissão. O oposto foi observado para prematuros tardios, nos quais as taxas de readmissão diminuíram conforme aumento do tempo de estadia hospitalar. A diferente associação entre o tempo de permanência hospitalar e o risco de readmissão, de acordo com a idade gestacional, pode ter duas explicações possíveis: a primeira é que a alta precoce é segura para bebês a termo, mas não para bebês prematuros tardios; a segunda é que a alta 
precoce é benéfica para $\mathrm{RN}$ a termo, mas isso não ficou claro no estudo devido ao viés relacionado à condição do bebê ao nascimento: um RN que necessita de maiores cuidados e, portanto, maior tempo de estadia, também estaria mais susceptível a reinternações (HARRON K, et al., 2017).

Outro estudo, realizado no Canadá, demonstra que o tempo de permanência hospitalar diminuiu entre os anos de 2003-2005 (dia 1: 41,2\%, dia 2: 40,8\%, dia 3: 11,9\%) e entre $2008-2010$ (dia 1: 50,9\%, dia 2: 35,8\%, dia 3: 8,7\%); já a taxa de readmissão aumentou 13\%, quando comparados os períodos de 2003-2005 e 20082010. Porém, o autor sugere que as taxas de reinternação aumentadas são devido à diminuição do limiar de internação, e não devido ao tempo de permanência, visto que as maiores taxas de reinternação foram observadas nos pacientes que apresentaram um tempo de permanência prolongado (cerca de 5-6 dias) (METCALFE A, et al., 2016).

Segundo Lain SJ, et al. (2015), as crianças que recebem alta hospitalar nos primeiros dois dias após o nascimento apresentam maiores chances de reinternação devido à icterícia do que as que tiveram alta após três dias. Essa associação diminui com o aumento da idade gestacional (LAIN SJ, et al., 2015).

Um melhor acompanhamento após a alta hospitalar por profissionais capacitados pode ser benéfico na redução das taxas de reinternação. Várias instituições argumentam, com base em evidências científicas, que a primeira consulta deve ser realizada de imediato, antes do fim da primeira semana de vida. Dentre essas instituições, a Academia Americana de Pediatria sugere, para todos os neonatos com alta antes de 48 horas, uma avaliação nas primeiras 48 horas após a alta, com o objetivo de estimular a amamentação e detectar icterícias ou outras manifestações de doenças possíveis de serem diagnosticadas nos primeiros dois dias de vida (ASENSI MONZÓ, et al., 2014; DELGADO MA e FURZÁN, 2014). No Brasil, o Ministério da Saúde estabelece que a primeira consulta após alta seja realizada com cinco dias (BRASIL, 2015).

A consulta com menos de 96 horas permite a detecção de desidratação hipernatrêmica no início e com níveis de sódio inferiores aos diagnosticados na consulta após uma semana. Em relação à amamentação, aqueles RN que receberam acompanhamento no sétimo dia de vida tinham 11,5 vezes mais chances de apresentarem problemas. Comparados aos que foram vistos em até 96 horas (ASENSI MONZÓ, et al., 2014).

\section{CONSIDERAÇÕES FINAIS}

Sendo assim, as evidências atuais reiteram a recomendação de que a alta do RN deve ser avaliada individualmente, observando-se os critérios de alta precoce, as condições clínicas da mãe e do lactente, o preparo da mãe pra cuidar do bebê e o acesso à assistência a saúde após a alta, diminuindo assim as chances de reinternação e as suas implicações. Além disso, com base na revisão realizada, percebe-se que no Brasil não existem estudos que avaliam o impacto da alta precoce na saúde do neonato.

\section{REFERÊNCIAS}

1. ASENSI MONZÓ M, et al. Atención en las primeras 48 horas tras el alta de maternidad en la consulta de enfermería o Pediatría. Pediatría Atención Primaria, v. 16, n. 62, p. 117-124, jun. 2014.

2. BENITZ WE. Hospital stay for healthy term newborn infants. Pediatrics, 135(5), 948-953, may 2015.

3. BRASIL. Ministério da Saúde. Portaria $n^{\circ}$ 2.068, de 21 de outubro de 2016. Institui diretrizes para a organização integral e humanizada à mulher e ao recém-nascido no Alojamento Conjunto. Diário Oficial da União, Brasília, DF, out. 2016.

4. BRASIL. Ministério da Saúde. Portaria nำ1.130, de 5 de agosto de 2015. Institui a Política Nacional de Atenção Integral à Saúde da Criança (PNAISC) no âmbito do Sistema Único de Saúde (SUS). Diário Oficial da União, Brasília, DF, 5 ago. 2016.

5. BRAVO P, URIBE C, CONTRERAS A, et al. Early postnatal hospital discharge: the consequences of reducing length of stay for women and newborns. Revista Da Escola De Enfermagem Da USP, v. 45, n. 3, p. 758-763, jun. 2011.

6. CHANG RKR, GURVITZ M, RODRIGUEZ S. Missed diagnosis of critical congenital heart disease. Archives of pediatrics \& adolescent medicine, 162(10), 969-974, oct. 2008.

7. DATAR A, SOOD N. Impact of post partum hospital-stay legislation on newborn length of stay, readmission, and mortality in California. Pediatrics;118(1):63-72, july. 2006. 
8. DE CAROLIS MP, et al. Individualized follow up programme and early discharge in term neonates. Italian Journal of Pediatrics, v. 40, p. 70, 15 jul. 2014.

9. DELGADO MA, FURZÁN JA. Alta neonatal precoz y consulta de seguimiento inmediato. Archivos Venezolanos de Puericultura y Pediatría, v. 77, n. 1, p. 4-8, mar. 2014.

10. HARRON K, et al. Newborn Length of Stay and Risk of Readmission. Paediatric and Perinatal Epidemiology, v. 31 , n. 3, p. 221-232, 2017.

11. JACKSON, GL, KENNEDY KA, SENDELBACH DM, TALLEY DH, ALDRIDGE CL, VEDRO DA, LAPTOOK AR. Problem identification in apparently well neonates: implications for early discharge. Clinical pediatrics, 39(10), 581590 , oct. 2000.

12. JAMES L, SWEET L, DONNELLAN-FERNANDEZ R, et al. Breastfeeding initiation and support: A literature review of what women value and the impact of early discharge. Women and Birth: Journal of the Australian College of Midwives, v. 30, n. 2, p. 87-99, abr. 2017.

13. KILPATRICK SJ, PAPILE LA, \& MACONES GA. Guidelines for perinatal care. American Academy of Pediatrics. 2017.

14. KURTH E, et al. Safe start at home: what parents of newborns need after early discharge from hospital - a focus group study. BMC health services research, v. 16, p. 82, mar. 2016.

15. LAIN SJ, et al. Early discharge of infants and risk of readmission for jaundice. Pediatrics, v. 135, n. 2, p. 314-321, fev. 2015.

16. LEMYRE B, JEFFERIES AL, O'FLAHERTY P. Facilitating discharge from hospital of the healthy term infant. Paediatrics \& child health, 23(8), 515-522, nov. 2018.

17. LUCIANO R. Early discharge of term neonates: we can do it safely. Italian journal of pediatrics. BioMed Central. Vol. 41, No. 2, p. A42, dec. 2015.

18. METCALFE A, et al. Proportion of neonatal readmission attributed to length of stay for childbirth: a population-based cohort study. BMJ open, v. 6, n. 9, p. e012007, 142016.

19. RITE GRACIA SR, et al. Criterios de alta hospitalaria del recién nacido a término sano tras el parto. Anales de Pediatría, v. 86, n. 5, p. 289.e1-289.e6, maio 2017.

20. SILVA, HMCPP. Alta precoce pós-parto: proposta de modelo hospitalar no Algarve (Master's thesis, Universidade de Évora), dec. 2017.

21. PAUL IM, LEHMAN EB, HOLLENBEAK CS, MAISELS MJ. Preventable newborn readmissions since passage of the Newborns and Mothers Health Protection Act. Pediatrics.;118(6):2349-235, dec. 2006.

22. WATT S, SWORD W, KRUEGER P. Longer post partum hospitalization options- who stays, who leaves, what changes? BMC Pregnancy Childbirth, oct. 2005;5:13.

23. ZIMMERMAN DR, KLINGER G, MERLOB P. Early discharge after delivery. A study of safety and risk factors. The Scientific World Journal, 3, 1363-1369, dec. 2003.

24. ZUPPA AA, et al. Critical issues in the early discharge of the newborn. (Napoli -- ITA, 12-15). Journal of pediatric and neonatal individualized medicine, oct. 2016. 